\title{
CT and COVID-19: Chinese experience and recommendations concerning detection, staging and follow-up
}

\author{
Li Fan $^{1} \cdot$ ShiYuan Liu ${ }^{1}$
}

Received: 30 March 2020 / Accepted: 15 April 2020 / Published online: 6 May 2020

(C) European Society of Radiology 2020

The outbreak of COVID-19 that started late December 2019, has spread to 184 countries on March 21, 2020, with 267,013 confirmed cases and 11,201 deaths according to the WHO reports [1]. China has accumulated huge clinical experience and reported about epidemiological, clinical, laboratory, and imaging features [2-6]. The National Health Commission of the People's Republic of China has published the 7th trial version of diagnosis and treatment of COVID-19 proposal [7].

Several suggestions and key points have been summarized in order to share this experience and maybe help teams that are now confronted with the outbreak worldwide.

\section{Should CT be performed for the initial diagnosis?}

The gold standard for diagnosis is RT-PCR. However, a high false negative rate has been reported [2], which would affect the timely management of suspected cases like isolation and medical treatment, further increasing the risk of persistent transmission. CT plays a major role in detecting pneumonia. The presence of viral pneumonia is one of the most important diagnostic criteria for the suspected cases. It has been reported that CT has high accuracy in reference to the RT-PCR [8], keeping in mind that the imaging features of COVID-19 pneumonia are non-specific, sometimes overlapping with other viral pneumonia [9-11]. If the patient has the epidemiological history, clinical features, and viral pneumonia features, he/she

Please note that due to the time sensitive nature of the work presented in this article, standard peer-review has been bypassed to ensure rapid publication. The article has been directly assessed by the Editor-in-Chief.

ShiYuan Liu

liushiyuan@smmu.edu.cn

1 Department of Radiology, Changzheng Hospital, Second Military Medical University, No. 415 Fengyang Road, Shanghai 200003, China should be considered probably positive for COVID-19 infection, despite negative or even second RT-PCR. Such patients should receive follow-up CT to evaluate the changes of pneumonia, and RT-PCT can be re-tested for three, four, or even five times $[8,12]$. Meanwhile, isolation needs to be applied to reduce the risk of transmission. In the fifth version of diagnosis and treatment of COVID-19 issued by the National Health Commission of the People's Republic of China [13], CT was considered a major modality for diagnosis, even before receiving the RT-PCR tests. Those clinically diagnosed case should be isolated and receive medical treatment. This should be considered with much attention by other countries if a huge population is waiting for the RT-PCR test, due to the lack of kits, delay waiting for the results, as well as false negative cases. $\mathrm{CT}$ can be considered a useful test for relieving quickly difficult situations.

\section{What is the balance between CT and RT-PCR?}

How to balance the role of $C T$ and RT-PCR, depending on the local prevention and control policy If the hospital has the possibility of performing RT-PCR, it should be performed more than twice if negative for patients with pneumonia on CT. According to the current guidelines, the suspected case would be excluded with the negative RT-PCR results twice within the interval of more than $24 \mathrm{~h}$. However, several reports showed the positive results occurred until the third, even the fifth, test $[8,12]$. Moreover, the mean interval time between the initial negative to positive RT-PCR results was $5.1 \pm 1.5$ days; therefore, the $24 \mathrm{~h}$ between the first two RT-PCT should be reconsidered. $C T$ findings are not always consistent with RT$P C R$, which is not surprising as CT and RT-PCR do not explore the same issues. Some patients with positive RT-PCR had normal CT $[2,6]$, but may later develop pneumonia, while others would not [6]. Some patients with negative RT-PCR results had marked viral pneumonia features on CT; some RT-PCR results would become positive after repeated tests with about a mean 
interval of 5 days [8]. In the largest cohort of 1014 cases from Wuhan, China, the sensitivity of chest CT imaging for COVID19 was $97 \%$ (580/601) with RT-PCR as a reference [8]. In patients with negative RT-PCR results but positive chest CT scans $(n=308$ patients), $48 \%$ (147/308) of patients were reconsidered as highly likely cases, with $33 \%(103 / 308)$ as probable cases by a comprehensive evaluation. The role of CT in this study was overestimated due to lack of the etiology and the prognosis analysis for those highly likely and probable cases.

\section{CT grading vs clinical outcome}

According to the 6th version proposal issued by China, COVID-19 is classified into four stages: mild, moderate, severe, and critical stages based on the severity of it. The mild stage shows absence of pneumonia. However, the presence and severity of pneumonia is not consistent with clinical stages. Clinically severe cases without pneumonia are observed in $5.2 \%$ [2]. In addition, clinical symptoms are not parallel to CT findings, as asymptomatic patients may have typical viral pneumonia features and positive RT-PCR [14]. These patients are obviously sources of contamination. Some cases with clinical improvement and turning negative RT-PCR still show pneumonia persistence, and even occurrence of new lesions [8]. Therefore, we need more investigation of the relationship between CT grading and clinical outcome. We expect CT grading to help evaluate severity and prognosis. Several CT grading or staging systems have been proposed $[4,15]$, based on the empirical descriptive system, just like in the case of lobar pneumonia, or based on the days of clinical symptom onset. The ideal CT grading and/or staging system should be based on pathological change, clinical symptoms, and imaging manifestation. The quantitative evaluation of pneumonia is required by the 7th version proposal [7], as the increase of 50\% pneumonia within $24-48 \mathrm{~h}$ is classified as a severe case; and the absorption and dissipation of pneumonia is also quantified during the follow-up scanning. Lobar-based quantitative analysis and scoring with qualitative analysis has been reported [16], but its relationship with different clinical stages and prognosis has not been evaluated.

\section{Should we perform follow-up CT during the acute phase and what are the recommended interval and doses?}

Rapid progression is one of the main characteristics of COVID-19. Imaging is the best modality to evaluate its progression. Chest radiography, especially bed side, is recommended for monitoring the very severe and critical cases. Conversely, CT is recommended for screening and followup of pneumonia due to high resolution. A dozen of studies showed different follow-up intervals from days 1 until 14, and displayed the rapid changes of imaging features, with progression into ARDS or resolution. Reported CT protocol included normal dose or low dose. For initial CT scanning, normal dose CT is recommended, because early imaging change is of small patchy ground glass opacity (GGO), which could be missed on low-dose CT. Conversely, low-dose CT is recommended for the follow-up considering the accumulation dose within a short term. The Chinese expert consensus recommends an interval of 3-5 days when initial CT was normal, and 5-7 days in case of pneumonia on the initial CT [15]. A shorter interval is recommended in case of normal initial CT, in order to evaluate the presence of pneumonia due to its rapid change. The interval of imaging follow-up depends on the severity, also guided by the idea of dose reduction.

\section{Which imaging after discharge?}

The discharge and/or remove isolation criteria in China [7] include normal body temperature at least 3 days, improvement of respiratory symptoms, improvement of the acute exudative imaging findings, and negative RT-PCR results twice with the interval of more than $24 \mathrm{~h}$. The imaging findings are subjectively appreciated, affecting reliability. Another question is the RT-PCR interval: 24 hay be too short and the high false negative rate should be considered as well. Recently, several cases which were previously discharged came up with positive RT-PCR again [17], indicating the necessity to reconsider the discharge criteria.

Due to CT with high sensitivity, follow-up CT is recommended to evaluate the improvement or recurrence on the first week of discharge, timely helping the management.

With the epidemic outbreak worldwide, more and more issues should be further evaluated, transforming the initially descriptive evaluation into systematic research. Quantitative imaging and big data combining the epidemiological, clinical, laboratory, and imaging features would be essential for improving diagnostic criteria, therapeutic evaluation, and prediction of outcome. Sharing clinical experience and data is imperative and would be a positive consequence of this dramatic outbreak.

Funding information This study has received funding from the National Natural Science Foundation of China (grant number 81871321), and National Key R\&D Program of China (2017YFC1308703, grant number 2016YFE0103000).

\section{Compliance with ethical standards}

Guarantor The scientific guarantor of this publication is Prof. Shiyuan Liu. 
Conflict of interest The authors of this manuscript declare no relationships with any companies whose products or services may be related to the subject matter of the article

\section{Statistics and biometry Not applicable}

Informed consent Not applicable

Ethical approval Not applicable

Methodology Not applicable

\section{References}

1. WHO. https://experience.arcgis.com/experience/ 685d0ace521648f8a5beeee1b9125cd. Accessed 21 Mar 2020

2. Guan WJ, Ni ZY, Hu Y et al (2020) Clinical characteristics of 2019 novel coronavirus infection in China. medRxiv. 2020.02.06.20020974. https://doi.org/10.1101/2020.02.06.20020974

3. Zhu N, Zhang D, Wang W et al (2020) A novel coronavirus from patients with pneumonia in China, 2019. N Engl J Med 382(8): 727-733. https://doi.org/10.1056/NEJMoa2001017

4. Jin YH, Cai L, Cheng ZS et al (2020) A rapid advice guideline for the diagnosis and treatment of 2019 novel coronavirus (2019$\mathrm{nCoV}$ ) infected pneumonia (standard version). Mil Med Res 7(1): 4. https://doi.org/10.1186/s40779-020-0233-6

5. Chen N, Zhou M, Dong X et al (2020) Epidemiological and clinical characteristics of 99 cases of 2019 novel coronavirus pneumonia in Wuhan, China: a descriptive study. Lancet. 395(10223):507-513. https://doi.org/10.1016/S0140-6736(20)30211-7

6. Lu H, Ai J, Shen Y et al (2020) A descriptive study of the impact of diseases control and prevention on the epidemics dynamics and clinical features of SARS-CoV-2 outbreak in Shanghai, lessons learned for the metropolis epidemics prevention. medRxiv. https:// doi.org/10.1101/2020.02.19.20025031

7. Diagnosis and treatment protocols of COVID-19 infection (trial version 7). The National Health Commission of the People's Republic of China [EB/OL]. http://www.nhc.gov.cn/yzygj/ s7653p/202002/8334a8326dd94d329df351d7da8aefc2.shtm1. Accessed 3 Mar 2020
8. Ai T, Yang Z, Hou H et al (2020) Correlation of chest CT and RTPCR testing in coronavirus disease 2019 (COVID-19) in China: a report of 1014 cases. Radiology. 26:200642. https://doi.org/10. 1148/radiol.2020200642

9. Shi H, Han X, Zheng C (2020) Evolution of CT manifestations in a patient recovered from 2019 novel coronavirus (2019-nCoV) pneumonia in Wuhan, China. Radiology:200269. https://doi.org/10. 1148/radiol.2020200269

10. Song F, Shi N, Shan F et al (2020) Emerging coronavirus 2019nCoV pneumonia. Radiology. 200274. https://doi.org/10.1148/ radiol.2020200274

11. Chung M, Bernheim A, Mei X et al (2020) CT imaging features of 2019 novel coronavirus (2019-nCoV). Radiology.:200230. https:// doi.org/10.1148/radiol.2020200230

12. Xie X, Zhong Z, Zhao W, Zheng C, Wang F, Liu J (2020) Chest CT for typical 2019-nCoV pneumonia: relationship to negative RTPCR testing. Radiology. 12:200343. https://doi.org/10.1148/ radiol.2020200343

13. Diagnosis and treatment protocols of COVID-19 infection (trial version 5). The National Health Commission of the People's Republic of China [EB/OL]. http://www.nhc.gov.cn/yzygj/ s7653p/202002/3b09b894ac9b4204a79db5b8912d4440.shtml. Accessed 4 Feb 2020

14. Bai Y, Yao L, Wei T et al (2020) Presumed asymptomatic carrier transmission of COVID-19. JAMA. https://doi.org/10.1001/jama. 2020.2565

15. Chinese Society of Radiology (2020) Radiological diagnosis in novel coronavirus pneumonia: expert recommendation from the Chinese Society of Radiology (First edition). Chin J Radiol 54(00):E001-E. https://doi.org/10.3760/cma.j.issn.1005-1201. 2020.0001

16. Bernheim A, Mei X, Huang $M$ et al (2020) Chest CT findings in coronavirus disease-19 (COVID-19): relationship to duration of infection. Radiology. 20:200463. https://doi.org/10.1148/radiol. 2020200463

17. Lan L, Xu D, Ye G et al (2020) Positive RT-PCR test results in patients recovered from COVID-19. JAMA. https://doi.org/10. 1001/jama.2020.2783

Publisher's note Springer Nature remains neutral with regard to jurisdictional claims in published maps and institutional affiliations. 